\title{
Excitation of Non-Radial Oscillations by Overstable Convection in Differentially Rotating Massive Main Sequence Stars
}

\author{
Umin Lee \\ Department of Astronomy, University of Tokyo, Tokyo 113, Japan
}

Summary.

We investigated overstable convective modes of differentially rotating massive main sequence stars. It is examined that the overstable convective modes in a rapidly rotating convective core may excite envelope non-radial oscillations by the resonance coupling between them. Let us denote by $q$ the ratio of the angular velocity of the rapidly rotating core to that of the envelope. Then, it is found that as the ratio $q$ increases, the order of the non-radial 8 modes resonantly coupled with the convective mode becomes lower, so that the excited $g$ modes come to have shorter periods in an inertial frame. It is also found that when the separation of the frequencies of the consecutive envelope 8 modes is wider than the resonance width, there occurs the alternation between the strong and the weak resonances, i.e., the resonantly coupled mode becomes overstable or neutral according to the strength of the resonance.

Our equilibrium model is a main sequence of $10 M_{o}$, which has a convective core of $3.25 M_{0}$. The model is assumed to rotate with velocity of about 110 $\mathrm{km} / \mathrm{sec}$ at the equator. Modes investigated are with $-m=l=2$. In this case, the shortest excited mode we obtain has periods of about a half day, in which the ratio $q$ is about 1.5 . We may conclude that if the differential rotation is assumed, the excitation by the overstable convection may give the widely applicable explanation to the early type star pulsations.

References

Lee, U. \& Saio, H. 1986, Mon. Not. R. astr. Soc., 221, 365.

Lee, U. \& Saio, H. 1987, Mon. Not. R. astr. Soc., 224, 513. 
Fig. 1
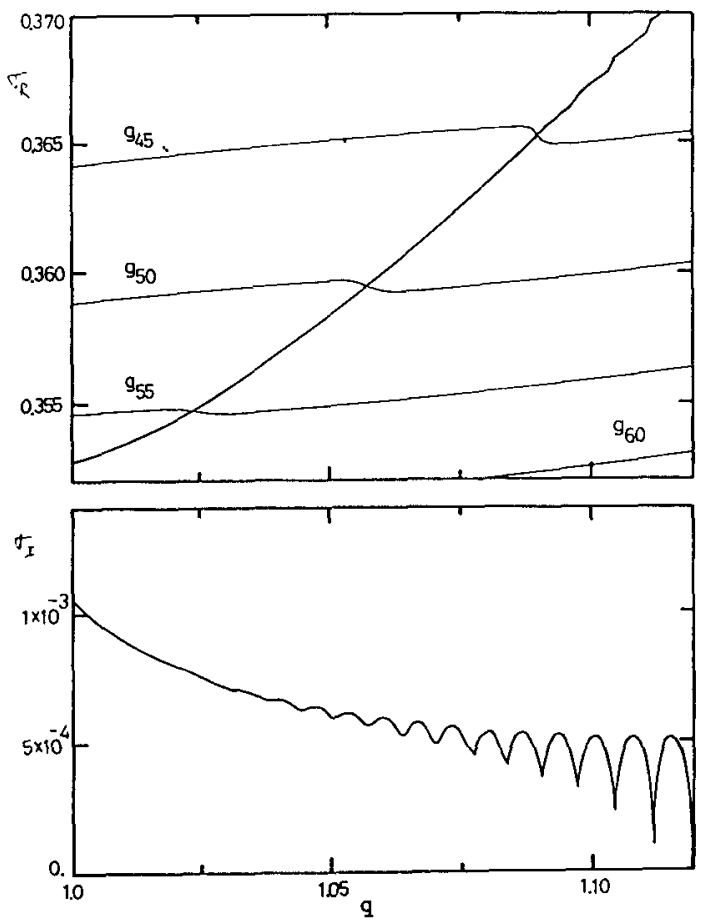

Fig. 1

Complex eigen-frequencies of overstable convective modes coupled with envelope $g$ modes as a function of the ratio $q$ (thick curves). Keal frequencies of high order envelope $g$ modes are also given (thin curves). kesonance couplings find themselves in the wavy features in the imaginary part of the frequency .

Fig. 2

Fig. 2

Complex eigen-frequencies of the convective mode coupled with envelope $g$ modes as a function of the ratio $q$. Feal frequencies of the envelope $g$ modes are also given. The thick curves denote the overstable modes and the thin curves neutral modes. The convective mode becomes overstable (complex freq.) or neutral (real freq.) according to the strength of the resonance.
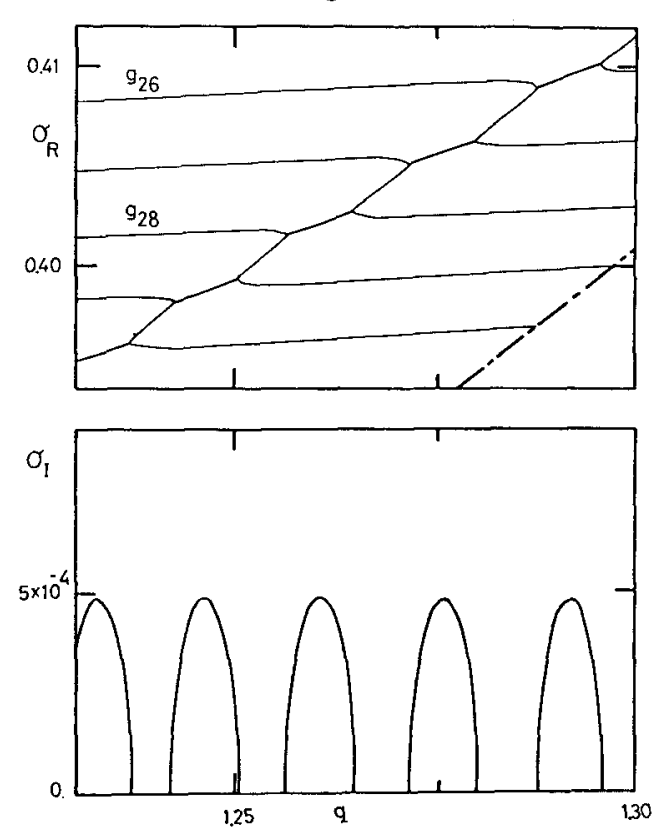